\title{
A Comparative Study of Different Techniques of Brachial Plexus Block in Upper Limb Surgery
}

\author{
Ahsan Mustafa ${ }^{1}$, Atif Mohammed ${ }^{2}$, Shaher Banu ${ }^{3}$ \\ ${ }^{1}$ Associate Professor, Anesthesiology, Deccan College of Medical Sciences, Hyderabad, India, ${ }^{2}$ Senior Resident, Anesthesiology, Deccan College of \\ Medical Sciences, Hyderabad, ${ }^{3}$ Post Graduate, Anesthesiology, Deccan College of Medical Sciences, Hyderabad, India.
}

\section{Abstract}

Background: Different techniques of Brachial Plexus block in upper limb surgery depending on the site of operation the approach of the brachial plexus was chosen. For the operations around the shoulder joint interscalene approach was chosen; for the operations up to the shoulder either interscalene or supraclavicular or Infraclavicular routes were chosen and for the operations up to and around the elbow joint any one of the four approaches were tried. Objective of this study was to evaluate its clinical applications and practical utility in providing good analgesia for the upper extremity surgery in elective as well as emergency cases. Design: This was a hospital based retrospective study. Duration: One Year i.e. from June 2018 to June 2019. Participants: 80 patients. Subjects and Methods: The present study includes 80 blocks of brachial plexus by four different routes. 1. Supraclavicular approach -20 cases. 2. Infraclavicular approach - 20 cases. 3 . Axillary perivascular approach -20 cases. 4 . Interscalene -20 cases. The cases were selected at random, who were subjected for various types of surgery of upper extremity both elective and emergency as in-patients. Results: In this series of 80 blocks, when the type of the surgery (emergency or elective) is considered, it has been that about one third of the patients underwent emergency surgery successfully with the brachial plexus block. In the remaining two-third of the patients, the surgery was planned procedure. Conclusion: Thus, our study reveals that the brachial plexus block can be equally safely employed for emergency surgery as for any elective procedure. Some-times the brachial plexus block can be the only choice with no alternatives affording a reasonable chance to a moribund patient to undergo emergency surgery. The dosage of the drug was modified according to the patient's general condition.

Keywords: Brachial plexus, supraclavicular, interscalene, Infraclavicular, Axillary.

Corresponding Author: Dr. Ahsan Mustafa, Associate Professor, Anesthesiology, Deccan College of Medical Sciences, Hyderabad, India. Email: drahsandcms@gmail.com

Received: January 2020

Accepted: January 2020

\section{Introduction}

Sherrington was the first person to elucidate that pain is a sensation comprised of a sensory and an effective component. From the recent studies a unified theory of the mechanisms of pain has evolved. It combines both physiologic and psychological aspects.

In the beginning, several crude methods were applied to alleviate pain during operation. Various methods and techniques have been developed in the course of time to give the surgeon the optimum conditions for surgery and provide the patient maximum possible pain relief . [1]

The object of an anesthesiologist is providing not only ideal conditions necessary for the surgery not only ideal conditions necessary for the surgery but also to minimize the undesirable effects before, during and after the administration of anesthesia.

For the operations involving upper extremities, the anesthetic techniques in use are, general anesthesia, intravenous regional anesthesia (I.V.R.A) and brachial plexus block.

Though, general anesthesia maintains its place as the method of choice in the great majority of patients, it is at times associated with physiological disturbances during and after the administration of anesthesia. There are several occasions, where one has to go in for different anesthetic techniques other than general anesthesia. These include emergency operations where the patients have had ingested food recently, patients with certain systemic organ diseases and in some inadvertent environments where general anesthesia is not possible. ${ }^{[2]}$

Intravenous regional anesthesia has its own limitations and drawbacks - viz: the drug can produce severe systemic toxic reactions due to rapid absorption into systemic circulation either because of improper application of the tourniquet or at the end of the surgery, after an early removal of the tourniquet. The intravenous regional anesthesia cannot be adopted in those cases where immobilization of the extremity in a plaster cast extending up to the region of application of the tourniquet. Hence for the surgery above the elbow intravenous regional anesthesia is not suitable. This technique is also not feasible for operations lasting for more than one hour. ${ }^{[3]}$

The brachial plexus block consists of injecting local 
analgesic solution in the fascial spaces surrounding the nerve plexus, thereby blocking the autonomic, sensory and motor fibers supplying the upper extremity. This procedure has been adopted with safety for many of the operations, involving the upper limb. This technique is simple and safe. In addition, the complications with this technique are minimal and the duration of hospitalization of the patients can be reduced.

The merits of regional anesthesia compared to general anesthesia are many and have been well documented. The pain relief during the perioperative period can be maintained in the postoperative period, reducing the occurrence of sideeffects caused by opioids (especially pruritus, nausea, vomiting and sedation). There are various approaches to the brachial plexus block such as Supraclavicular, Interscalene, Infraclavicular and Axillary approaches. ${ }^{[3,4]}$

Brachial plexus block can under certain circumstances be the only choice affording a reasonable chance to a moribund, patient to undergo surgery. In daily busy schedules, time factor may limit the relatively time-consuming brachial plexus block as compared to general anesthesia and intravenous regional anesthesia, but might be the only choice in certain cases. ${ }^{[5]}$

\section{Aim of the study: brachial plexus - viz; \\ 1) Supraclavicular approach. \\ 2) Infraclavicular approach \\ 3) Axillary approach \\ 4) Interscalene approach}

Aim of this study was to evaluate its clinical applications and practical utility in providing good analgesia for the upper extremity surgery in elective as well as emergency cases.

In this study, the four different approaches to block the

These have been made and an attempt has also been made to formulate the advantages and disadvantages of the four routes.

Finally, the complications, both immediate as well as late of the brachial plexus block were also analyzed.

\section{Subjects and Methods}

Place of Study: Owaisi Hospital, Hyderabad,

Sampling Methods: The present study includes 80 blocks of brachial plexus by four different routes

1) Supraclavicular approach - 20 cases

2) Infraclavicular approach - 20 cases

3) Axillary perivascular approach -20 cases

4) Interscalene - 20 cases.

The cases were selected at random, who were subjected for various types of surgery of upper extremity both elective and emergency as in-patients.

\section{Inclusion Criteria}

- Patients of both sexes aged 20 years \& above up to 65 years of age, who were posted for operation of the upper extremity were selected. Patients below the age of 20 years were not selected for this procedure, as they were usually non co-operative. Patients above the age of 65 years were also not selected because of the associated geriatric problems.

- Patients who had fever, severe systemic diseases and neurological complications and patients who were mentally deficit were avoided from the study.

- Patients with sepsis at the site of block were not selected.

- Patients who gave history of allergy and hypersensitive manifestations to drugs were not selected.

- Patients who were nervous and who refused to have local blocks were also not selected.

- Cases where the proposed surgery was lasting for not more than 30 minutes were not selected for the study.

- Patients undergoing elective and emergency procedures were also included.

Choice of the route of the brachial plexus block:

- Depending on the site of the operation, the approach of the brachial plexus block was chosen, as follows:

- For the operations around the shoulder joint, interscalene approach was chosen, for the obvious reason of obtaining the higher level of analgesia.

- For the operations up to the shoulder either interscalene or Infraclavicular or supraclavicular approaches were chosen.

- For the operations below the shoulder joint, any of the four approaches were chosen.

- Informed written consent was taken from the study participants or from the blood relatives in case of emergency situations prior to the start of the study.

\section{Exclusion Criteria}

Those who were not willing to give consent or participate in the study

\section{Statistical analysis:}

- Data entry was done using Microsoft excel 2010 version and analysis using EPI INFOR version 7. Data was presented in percentages and proportions.

\section{Results}

After about 10 minutes of the administration of the block, onset of sensory loss was tested with pin pricks and the time of the onset of sensory loss was noted. The surgeon was allowed to proceed with his surgery after the establishment of sensory loss.

In all the cases, where the block was failed partially or completely, then general anesthesia was supplemented.

The patient's blood pressure, pulse and respirations were monitored during the surgery.

When the action of the brachial plexus block appeared to wear off and the surgery was to be continued, then general anesthesia was supplemented.

The onset of the recovery of the sensory block was noted.

Post-operative follow-up for 24 hours in all the cases was made to note for any complications due to the brachial plexus block.

The present study of Brachial plexus block for upper extremity surgery was undertaken in a series of 80 cases in Owaisi Hospital, Hyderabad, from 2014-2016.

\section{The routes of approach employed in this series were:}


1) Supraclavicular approach.

2) Infraclavicular approach

3) Axillary approach

4) Interscalene approach.

The observations were analyzed in different tables as shown:

Table 1: Table Showing Sex Wise and Block Wise Distribution of Cases

\begin{tabular}{|l|l|l|l|l|}
\hline Sl.No. & Approach & Male & Female & Total \\
\hline 1 & Supraclavicular & 16 & 04 & 20 \\
\hline 2 & Infraclavicular & 17 & 03 & 20 \\
\hline 3 & Axillary & 18 & 02 & 20 \\
\hline 4 & Interscalene & 14 & 06 & 20 \\
\hline & Total & 65 & 15 & 80 \\
\hline
\end{tabular}

I. The maximum number of blocks was performed for males $(81.2 \%)$ when compared to females

II. Maximum number of blocks was performed for males in Axillary route and for females in interscalene route as the allocation of patients for each group was random.

Table 2: Table Showing age Distribution in Each Route

\begin{tabular}{|l|l|l|l|l|l|}
\hline SI.No. & Approach & $\begin{array}{l}\mathbf{2 1 - 4 0} \\
\text { years }\end{array}$ & $\begin{array}{l}\mathbf{4 1 - 6 0} \\
\text { years }\end{array}$ & $\begin{array}{l}\mathbf{6 1 - 6 5} \\
\text { years }\end{array}$ & Total \\
\hline 1 & Supraclavicular & 18 & 02 & Nil & 20 \\
\hline 2 & Infraclavicular & 18 & 01 & 1 & 20 \\
\hline 3 & Axillary & 18 & 02 & NIL & 20 \\
\hline 4 & Interscalene & 14 & 04 & 2 & 20 \\
\hline & Total & 68 & 09 & 03 & 80 \\
\hline
\end{tabular}

The maximum numbers of blocks were performed between 21 - and 40-years age group (85\%).

Maximum number of blocks was performed for elective cases $(66.2 \%)$.

For emergency cases, maximum number of blocks was performed by supraclavicular approach.
Table 3: Table Showing Emergency and Elective Cases

\begin{tabular}{|l|l|l|l|l|}
\hline Sl.No. & Approach & Emergency & Elective & Total \\
\hline 1 & Supraclavicular & 11 & 09 & 20 \\
\hline 2 & Infraclavicular & 06 & 14 & 20 \\
\hline 3 & Axillary & 05 & 15 & 20 \\
\hline 4 & Interscalene & 05 & 15 & 20 \\
\hline & Total & 27 & 53 & 80 \\
\hline
\end{tabular}

Table 4: Table Showing the Onset of Sensory Blockade, Duration of Operation and Duration of Blockade (Analgesia).

\begin{tabular}{|l|l|l|l|l|}
\hline S.No. & Approach & $\begin{array}{l}\text { Onset of } \\
\text { sensory } \\
\text { blockade }\end{array}$ & $\begin{array}{l}\text { Duration } \\
\text { of } \\
\text { operation }\end{array}$ & $\begin{array}{l}\text { Duration } \\
\text { of } \\
\text { blockade }\end{array}$ \\
\hline 1 & Supraclavicular & 20 minutes & $21 / 4$ Hours & $21 / 2$ hours \\
\hline 2 & Infraclavicular & 21 minutes & 2 hours & 2 hours \\
\hline 3 & Axillary & 18 minutes & $11 / 4$ Hours & 2 hours \\
\hline 4 & Interscalene & 22 minutes & 2 Hours & $21 / 2$ hours \\
\hline
\end{tabular}

Among the four approaches, onset of sensory blockade was fastest in axillary approach (18min) followed by supraclavicular (20min). Duration of blockade was highest in supraclavicular and Interscalene approaches (2 $1 / 2$ hours) hours.

The success rate of the block was found to be highest by supraclavicular route $(92 \%)$. The rate of success by axillary route was next in order (84\%), then Infraclavicular (80\%) and the success rate was found to be least by the interscalene approach $(76 \%)$.

The block was found to be unsuccessful in $8 \%$ of the supraclavicular, $16 \%$ of the axillary, $20 \%$ of the infraclavicular and $24 \%$ of the interscalene routes.

The average percentage of success by all the four routes was found to be $82.5 \%$. The average percentage of failure by all the four routes was found to be $17.5 \%$.

Table 5: Table Showing the Success and Failure Rates of All the Four Blocks

\begin{tabular}{|c|c|c|c|c|c|c|c|c|c|}
\hline \multirow{2}{*}{$\begin{array}{l}\text { Sl. } \\
\text { No }\end{array}$} & \multirow[t]{2}{*}{ Approach } & \multicolumn{3}{|c|}{ Success Rate } & \multirow[t]{2}{*}{$\%$ success } & \multicolumn{3}{|c|}{ Failure rate } & \multirow[t]{2}{*}{$\%$ failure } \\
\hline & & $\mathbf{M}$ & $\mathbf{F}$ & $\mathbf{T}$ & & $\mathbf{M}$ & $\mathbf{F}$ & $\mathbf{T}$ & \\
\hline 1 & Supraclavicular & 14 & 04 & 18 & $92 \%$ & 02 & -- & 02 & $8 \%$ \\
\hline 2 & Infraclavicular & 14 & 02 & 16 & $80 \%$ & 03 & 01 & 04 & $20 \%$ \\
\hline 3 & Axillary & 16 & 01 & 17 & $84 \%$ & 02 & 01 & 03 & $16 \%$ \\
\hline \multirow[t]{3}{*}{4} & Interscalene & 10 & 05 & 15 & $76 \%$ & 04 & 01 & 05 & $24 \%$ \\
\hline & Total & 54 & 12 & 66 & $82.5 \%$ & 11 & 03 & 14 & $17.5 \%$ \\
\hline & $\mathrm{M}=$ Male & \multicolumn{3}{|c|}{$\mathrm{F}=$ Female } & & \multicolumn{3}{|c|}{$\mathrm{T}=$ Total } & \\
\hline
\end{tabular}

Table 6: Table Showing the Site of Operation

\begin{tabular}{|l|l|l|l|l|l|l|}
\hline Sl No. & Site of Operation & Supra clavicular & Infra clavicular & Axillary & Inter scalene & Total \\
\hline 1 & Around the shoulder joint & Nil & Nil & Nil & 01 \\
\hline 2 & Around the shaft of the humerus & 02 & 03 & Nil & 03 \\
\hline 3 & Around the elbow joint & 02 & 03 & Nil & 02 \\
\hline 4 & Around the shaft of radius or ulna & 05 & 03 & 04 & Nil & 04 \\
\hline 5 & Around the wrist joint & 08 & 03 & 03 & 04 & 02 \\
\hline 6 & Around the hand & 20 & 07 & 20 & 17 & 08 \\
\hline & Total & 20 & & 11 \\
\hline
\end{tabular}

Table 7: Table Showing the Immediate Complications I.E, While Administering the Block or During the Effective Period of the Block

\begin{tabular}{|l|l|l|l|l|l|l|}
\hline SI No. & Nature of complication & Supraclavicular & Infra clavicular & Axillary & Inter scalene & Total \\
\hline 1 & $\begin{array}{l}\text { Accidental puncture of a } \\
\text { vessel }\end{array}$ & 2 & 1 & 5 & - & 8 \\
\hline 2 & Drug toxicity & - & - & - & - & - \\
\hline 3 & $\begin{array}{l}\text { Pleural puncture, } \\
\text { pneumothorax }\end{array}$ & - & - & - & - & - \\
\hline 4 & Horner's syndrome & - & - & - & - & - \\
\hline & Total & 2 & 1 & 5 & - & - \\
\hline
\end{tabular}


Table 8: Table Showing the Late Complications of the Block I.E, Those Which Developed Post-Operatively Upto 24 Hours.

\begin{tabular}{|l|l|l|l|l|l|l|}
\hline SI No. & Nature of complication & Supraclavicular & Infra clavicular & Axillary & Inter scalene & Total \\
\hline 1 & Hematoma & - & - & 1 & - & - \\
\hline 2 & Pneumothorax & - & - & - & - & - \\
\hline 3 & Phrenic nerve palsy & - & - & - & - & - \\
\hline 4 & Brachial plexus injury & - & - & - & - \\
\hline 5 & Recurrent laryngeal nerve palsy & - & - & - & - & - \\
\hline 6 & Any other complications & - & - & - & - & - \\
\hline & Total & - & - & 1 & - & - \\
\hline
\end{tabular}

Maximum number of operations done was around the hand $(50 \%)$. In the supraclavicular \& infraclavicular route operations were done up to and around the shaft of the humerus. In the axillary route maximum operations were done around the hand. In the interscalene route, though figure is less, still it can be stated that operations can be done up to and around the shoulder joint.

The rate of immediate complications of the brachial plexus block was found to be highest by Axillary approach (25\%). The rate of complications by supraclavicular approach was next in order (10\%), Infraclavicular approach $(5 \%)$ and nil by interscalene approach. In total the rate of immediate complications by all the four routes was found to be $(10 \%)$.

An accidental puncture of vessel was found in the Axillary approach, Infraclavicular and in the supraclavicular approach. No other complications in the four approaches.

It was found that there was a rise in blood pressure by about $10 \mathrm{~mm}$ of $\mathrm{Hg}$ in most of the cases who received the brachial plexus block. This change appeared after the injection of the anesthetic agent and returned to the basal level, as the action of the drug started wearing off.

There was no change in respiration in any of the cases in our series.

Among the late complications of the brachial plexus block, only formation of hematoma of one case was encountered and that too in the axillary route. No late complications were seen in the other four routes.

\section{Discussion}

In the present study of 80 brachial plexus block, 20 blocks were performed by supraclavicular route, 20 by infraclavicular route, 20 blocks by axillary route and 20 blocks by interscalene route.

\section{Age Distribution}

The maximum number of blocks was performed in the age group of 21 and 40 years. Various other workers have successfully administered the brachial plexus block at the extreme of age and have achieved good results, Viz-accardo and Adriani in the year 1940 published their series of brachial plexus block with the minimum age being 1 year and the maximum age being 60 years. In the study of Turner and Clayton (1959-60), the age ranged between 6 weeks to 10 years. In the year 1962, D.C. Moore reported the minimum age in his study as 3 years and maximum as 60 years.

\section{Role of Sex of the Patient:}

In our series when the sex of the patient is taken into consideration, it appears that the sex of the patient has got no role to play either in the administration or in the success rate of the block.
Inpatient and Out-Patient Distribution:

In the present study of 80 blocks, all cases treated as inpatients. The brachial plexus block can be also employed safely as an Out-patient procedure.

\section{Type of the surgery (elective or emergency):}

In this series of 80 blocks, when the type of the surgery (emergency or elective) is considered, it has been that about one third of the patients underwent emergency surgery successfully with the brachial plexus block. In the remaining two-third of the patients, the surgery was planned procedure. Thus, our study reveals that the brachial plexus block can be equally safely employed for emergency surgery as for any elective procedure. Some-times the brachial plexus block can be the only choice with no alternatives affording a reasonable chance to a moribund patient to undergo emergency surgery. The dosage of the drug was modified according to the patient's general condition.

\section{Premedication:}

The patients in all the four approaches of the brachial plexus block were premedicated with injection midazolam $1 \mathrm{mg}$ intravenously $10 \mathrm{~min}$ prior to the administration of the block. The main object of the employing the premedication was twofold; the first one was for sedating the patient, so that the patient will be quiet and will extend full co-operation for performing a successful block. Secondly it was supposed to counteract the untoward actions of the inadvertent entry of the lignocaine into a vessel.

Rhone T.S. (1935) gave morphine and barbiturate as premedication. Patrick J. (194)) used intramuscular morphine $(10 \mathrm{mg})$ with injection atropine $(0.6 \mathrm{mg})$ to his series of patients.

\section{Anaesthetic agent used:}

In this study the local anesthetic agent employed was lignocaine $1.5 \%$ solution with Adrenaline 1:80,000 in an average dosage of $20 \mathrm{~m}(300 \mathrm{mg})$. The duration of the block lasted for about two hours and fifteen minutes.

Lignocaine $1.5 \%$ was used as an anesthetic agent in this study because it is freely available, most stable and least toxic drug. Moreover, it can be autoclaved repeatedly without loss of its potency and it has a very quick onset of action as it spreads easily through tissues and penetrates the nerve fibers because of its excellent power of diffusion. It is not a vasodilator nor does it interfere with the vasoconstriction action of adrenaline (Wylie and Churchill Davidson. 2007).

In this study to prolong the duration of action, adrenaline 1:80,000 was mixed with the anesthetic agent. The duration of action lasted for 2 hours to $2 \frac{1}{2}$ hours. When adrenaline was mixed with the anesthetic agent it causes local 
vasoconstriction of the blood vessels, thereby delays the absorption of the drug and leaves the drug to be in contact with the nerves for a longer time and thereby prolongs the duration of action of the drug. This also reduces the toxicity of the drug (Wylie and Churchill Davidson, 2007).

Bonica J.J. and Moore, D.C. (1950), used procaine 2\%, 40 $\mathrm{ml}$ to prolong the effect they used Pantocaine $0.15 \%, 40 \mathrm{ml}$ and metacaine $1.5 \%, 30 \mathrm{ml}$, the effect lasted for 1 hour 10 minutes with procaine, 1 hour 31 minutes with Pantocaine, and 1 hour, 56 minutes with metacaine.

\section{Onset and duration of analgesia:}

In our study in all the four approaches the onset of sensory loss varied between 15 minutes to 25 minutes, so the average onset of action of the brachial plexus block immaterial of the approach chosen can be taken as 20 minutes. The reason for this delayed onset of action is quite obvious i.e., the addition of adrenaline to the anesthetic solution.

When the duration of analgesia was observed, it was found to be between 2 hours and $2 \frac{1}{2}$ hours, irrespective of the approach. Thus, the average duration of analgesia of the brachial plexus block can be taken in our study as 2 hours and 15 minutes. These results closely resemble those of Kundu, J.P. (1970) who used lignocaine $1 \%$ with adrenaline 1 in 200,000 dilution and noted the onset to be 15 minutes and duration of analgesia lasting for 2 hours.

\section{Site and nature of operation:}

In this study of 80 blocks the various sites and natures of operation in both elective as well as emergency cases can be arbitrarily divided into those around the shoulder joint, the shaft of numerous, the elbow joint, the shaft of radius of ulna, around writs joint and those of the hand.

In the first group of 20 patients who received the brachial block by supraclavicular route, it can be seen that this approach can be successfully administered to the operations at any site of the upper extremity.

In the second group who had received the block by infraclavicular approach, it can be stated with confidence that this approach is mainly advocated for operations at any site of the upper extremity.

In the third group who had received the block by axillary approach, it can be stated with confidence that this approach is mainly advocated for operations around and below the elbow joint.

When the fourth group of 20 patients who were administered the brachial plexus block by interscalene route, it is seen that the percentage of the operations around shoulder joint was maximum in this group, and at the same time this interscalene approach was as safe other two methods for surgery at other sites of the upper extremity also.

Out of the four approaches the interscalene approach is the newer one which was described for the first time by Winnie. A.P.,6 in the year 1970. He states that interscalene approach is not intended to replace the axillary or supraclavicular technique. It is rather intended to provide a simple safe technique for extending the usefulness of perivascular anesthesia, to include surgery and/or manipulations of the upper arm, shoulder and even neck without the need to use inordinately large volumes. In addition, it offers an alternate technique for providing anesthesia for hand, upper arm and force arm surgery, when the axillary and supraclavicular techniques are impossible or contra-indicated due to infection or trauma in the areas.

The success rate of supraclavicular route was $92 \%$, the infraclavicular route was $80 \%$, the axillary route was $84 \%$ and interscalene route being $76 \%$. The average percentage of success by all the four routes was found to be $82.5 \%$.

For the purpose of discussion, we have attempted to classify the complications into 'immediate' and 'late'. The immediate complications include those occurring either during the administration of the block, or during the effective period of the block. The complications which occurred after the effective period of the block up to 24 hours were taken as late complications. Out of 80 brachial plexus blocks, 7 cases had immediate complications, they are, 7 cases of accidental puncture of a vessel. The late complication encountered was only one case of hematoma.

When the advantage and disadvantage of each route were analyzed, it was found that each route has its own merits and demerits. But in the supraclavicular approach the advantages outweighed the disadvantages and hence, it was concluded that, among the four routes which are in common use to block the brachial plexus, supraclavicular route is preferred to the other routes for all type of operations of the upper extremity, in all age groups, because of its ease of administration and minimal incidence of complications.

\section{Success rate \& failure rate supraclavicular route:}

Out of the 20 cases, the block was successful in 18 cases, and failed in the remaining 2 cases. Out of the 2 cases in which the block was failed, when the cause of the failure was analyzed - it has been found that there was aspiration of blood into the syringe while administering the anesthetic solution in 2 cases. In these 2 cases, the needle was withdrawn and the direction as well as position of the needle was changed and the block was attempted again. Probably altered direction and position of the needle in wrong place be the cause of failure in these 2 cases.

\section{Infraclavicular route:}

Out of the 20 blocks, failure was seen in 4 patients. The success rate of Infraclavicular route was $80 \%$.

Similar results were observed by Chandrasekaran Ayyavu et al (2016), ${ }^{[7]}$ on a comparative study of brachial plexus using Infraclavicular and axillary approaches in forearm surgery where time taken to perform block, successful blockade, tourniquet tolerance was better in infraclavicular approach group when compared to axillary group. Onsets of both sensory and motor blockade were similar in both the groups. Complication like vascular puncture was found to be more with axillary block than with infraclavicular block.

\section{Axillary route:}

In this series of 20 blocks, the failure was in 3 patients. This increased failure rate in the axillary approach as compared to supraclavicular approach can be because of Axillary vessels puncture and wrong placement of the needle. Hence the aspiration of blood was seen as in the supraclavicular route. However, out of the 3 failures, two failures are definitely because of the puncture of the vessels during administration and wrong placing of the needle subsequently. In the 
reaming one patient, in whom the block failed, the needle tip might have gone beyond the perivascular sheath, penetrating both the walls of the sheath.

\section{Interscalene route:}

In this third group of 20 patients, the success rate was found to be minimum i.e. (76\%) The block failed in 5 patients. The failure might be due to wrong placement of needle and the solution might not diffuse in sufficient quantity. This could be the cause of failure in our patients. Winnie (1970) advocates that this disadvantage of interscalene route can be overcome, by using a larger volume of the solution.

Study by Susmita Bhattacharyya et al $(2015),{ }^{[8]}$ observed that the onset of sensory and motor block was earlier in the supraclavicular group than the interscalene group but there was no sparing of any dermatome in the interscalene group. The duration of sensory block was significantly prolonged in the supraclavicular group but there was no difference regarding the duration of motor block among the two groups. Incidence of vessel puncture and Horner's syndrome were more in supraclavicular and interscalene group respectively. Our results are less compared with the study of Winnie, A.P. (1970), who claimed the success rate as $94 \%$ of the first 200 patients.

The immediate complications include those occurring either during, the administration of the block or during the effective period of the block. The complications which occurred after the effective period of the block up to 24 hours were taken as late complications. In our study we didn't encounter any of the early complications

- Accidental vessel puncture

- Pleural puncture

- Horner's Syndrome

\section{Accidental puncture of a vessel:}

The commonest complication encountered in our series is the accidental puncture of a vessel, while the needle was being advanced to the proper depth. Totally we had 7 cases of accidental puncture of a vessel in all the four routes, five being in the Axillary group, two in the supraclavicular group and none in the interscalene group. Incidence of highest number of this complication in Axillary group is because; the nerves from the brachial plexus and the Axillary vessels are enclosed in a fibrous neuromuscular fascial sheath. In the supraclavicular approach too, we have come across 2 cases of accidental puncture of a vessel, because of the situation of the plexus in the immediate vicinity of the great vessels. The reduce incidence of this complication in the supraclavicular approach as compared to Axillary route, is analyzed, it can be said that the relation of the vessel play an important part in the incidence of this complication. The relation of the axillary vessels is obviously closer than the subclavian vessels and the direction of the needle in the supraclavicular approach is approximately parallel to the direction of the subclavian vessels, where as it is nearly perpendicular in the axillary approach. Hence the puncture of vessels is more common in the axillary approach. In the interscalene approach the puncturing of a vessel is virtually impossible because of the situation of the brachial plexus away from the vessels (winnie, A.P. 1970)

In our series too, we have not come across this complication in interscalene approach. However, Winnie (1970) warns to be cautious in directing the needle in the interscalene approach as the horizontal direction of the needle may strike the vertebral artery.

\section{Pleural puncture:}

In our series, we have not come across pleural puncture in supraclavicular group. When the tip of the needle comes into contact with the parietal layer of the pleura; the patient will have a violent cough. The possibility of pneumothorax is always a bugbear in the supraclavicular approach. This complication has been observed by many previous workers ever since the brachial plexus block was introduced into clinical practice. This is also more common on the right side due to high level of the dome of the pleura and lung. This complication is more in pediatrics where the dome of the pleura and lung is high in the neck and to the presence of supraclavicular fat also hinders in the proper land mark. The older age group above 65 years will be usually suffering from some chronic respiratory diseases with emphysematous changes in the lungs and so the dome of the pleura and lungs are in a higher level in the neck. Hence, the complication of pneumothorax is more in these cases.

In the present study of 20 cases of supraclavicular approach the technique of Patrick's technique has been employed. Moreover, patients below 20 years and above 65 years have not been selected for study. Hence there is a definite reduction in the incidence of this complication.

The question of pleural puncture and pneumothorax does not arise in axillary as well as in interscalene approaches because of the obvious reason of the site being away from the dome of the pleura.

\section{Horner's syndrome:}

In our series of 75 brachial plexus block, no cases of Horner's syndrome have occurred. Horner's syndrome results from paralysis of the cervical sympathetic nerves to the eye. It is evidenced by: a) Constriction of the pupil (b) narrowing of the palpebral fissure (c) Apparent recession of the eye ball - enophthalmos (d) Frequently a reddening of the conjunctiva due to dilatation of its blood vessels from paralysis of their vasoconstrictive supply.

Horner's syndrome is more common in the supraclavicular approach because of the spillover of the anesthetic agent over the cervical sympathetic chain or stellate ganglion, as the administration of the anesthetic solution is just by the side of these structures and diffusion to these structures being easier. Sometimes the signs of a stellate ganglion block may also be encountered, if the spillover takes place more over the stellate ganglion. In those cases, patients may complain of stuffiness of the nasal mucosa, anhidrosis of the face and vasodilatation of the face and upper limb, all symptoms being exhibited on the side of the block.

This complication is virtually impossible in the axillary route. Again, chances of this complication recurring in the interscalene route are very much remote. The cervical sympathetic truck is situated medial to the vertebral artery and so when the direction of the needle is horizontal and pushed too much medially after striking the vertebral artery, block and Horner's syndrome may occur. 


\section{Other complications:}

Fortunately, we did not encounter any of the other immediate complications - viz: drug toxicity, hypotension and hypertension.

\section{Late Complications:}

Hematoma formation, pneumothorax, phrenic nerve palsy, brachial plexus injury causing residual palsy and recurrent laryngeal nerve palsy. Fortunately, we did not have any of the above said late complications in any of the routes, except a case of hematoma formation in the axillary approach group K.Whiffler et al (1981), ${ }^{[9]}$ conducted a prospective randomized controlled trial of Infraclavicular block using coracoid approach. They found that compared with supraclavicular approach pulmonary complications do not occur and also a higher level of analgesia can be obtained when compared to axillary approach.

J.Desroches et al (2003), ${ }^{[10]}$ conducted a study to describe the sensory distribution, motor blockade and clinical efficacy of infraclavicular approach of brachial plexus block by coracoid approach. They concluded that this approach of infraclavicular block has very good tolerance to tourniquet and produces extensive sensory blockade and consistent anesthesia for surgeries of the upper limb.

K J Chin et al (2013), ${ }^{[11]}$ conducted a study to compare other approaches of the brachial plexus with infraclavicular approach in terms of safety and efficacy. They concluded that for lower arm surgeries infraclavicular block provides efficient anesthesia like other techniques and is also simple to learn and perform. Also, tourniquet pain and discomfort during block is very less.

M Neuburger et al (1998), ${ }^{[12]}$ conducted a clinical trial on efficiency of vertical approach of infraclavicular block for providing brachial plexus anesthesia with use of peripheral nerve stimulator and found that complications like nerve lesions or pneumothorax does not occur. The vertical approach using nerve stimulator is an easy and simple technique for providing brachial plexus anesthesia and very easy to master.

HG Kilka, P Geiger et al (1995), ${ }^{[13]}$ found that infraclavicular brachial plexus block using vertical approach is a successful technique when compared to other techniques. It also provides excellent tourniquet tolerance of the upper arm for longer period of time. Onset of complete nerve block is achieved faster and this technique also provides longer period of analgesia postoperatively. Lower risks and high patient acceptance make this technique a better one.

$\mathrm{Z}$ Ertung et al (2005), ${ }^{[14]}$ conducted a clinical trial on comparison of axillary approach and infraclavicular approaches of brachial plexus blockade for surgeries involving forearm and arm and concluded that both the approaches are similar in terms of safety and efficacy. But infraclavicular approach is preferred to the axillary approach in terms of mobility.

Deleuze et al (2003), ${ }^{[15]}$ compared single stimulation lateral infraclavicular block with triple stimulation axillary block. The complete block in median, radial, ulnar,

Musculocutaneous, and medial antebrachial cutaneous nerves was taken for clinical success rate and they concluded that single shot infraclavicular block is equally effective as a triple nerve stimulation axillary block.

\section{Conclusion}

The present study consists of 80 brachial plexus blocks performed at Owaisi Hospital, Hyderabad with the cooperation of surgical and its ancillary, Departments to which our cases belonged. The study revealed the following conclusions.

- Brachial plexus block can be performed for both emergency and elective cases of both sexes, where general anesthesia is contraindicated or if the regional anesthesia is preferred.

- This technique is simple, economical and could be employed with leased equipment. But always-resuscitative equipment must be on hand.

- This can be employed to any type of operation and/or manipulations of the upper extremity.

- Lignocaine has hardly shown any sensitivity reaction as observed by performing an intradermal test done in every patient.

- Lignocaine $1.5 \%$ solution with Adrenaline 20 to $30 \mathrm{ml}$. (300 - $450 \mathrm{mg}$ ) was quite sufficient to block the plexus. The total dosage of the drug used was within the toxic dose.

- The onset of analgesia was also quite quicker and duration lasted for about 2 hours and fifteen minutes, which was quite sufficient for most of the operations.

- Using lignocaine with adrenaline, the action of lignocaine will be prolonged as well as toxicity of the lignocaine will be minimized. There were no toxic reactions noted to the adrenaline.

- The pre-operative preparation of the patient is very minimal and no need for starving the patient either preoperatively or post operatively.

- Among the four routes which are in common use for brachial plexus block, supraclavicular route is preferred to the other routes for all types of operations of the upper extremity, in all age groups, because of its ease of administration and minimal incidence of complications.

\section{References}

1. O' Alessio JG, Rosenblum M, Shea KP, et al: A retrospective comparison of interscalene block and general anesthesia for ambulatory surgery - shoulder arthroscopy Anesthesia. 1995;20:62-68.

2. Joseph M. Neal, James R. Hebl, J. C. Gerancher, Quinn H. Hogan. Brachial plexus Anesthesia: Essentials of our current understanding. Regional anesthesia and pain medicine. 2002;27:402-428.

3. Tran Q, Clemente A, Doan J, Finlayson R. Brachial plexus blocks: a review of approaches and techniques. Can J Anesth/J Can Anesth. 2007;54:662-674.

4. Yang C, Kwon H, Cho C, Jung S, Kang P, Park E et al

5. A comparison of infraclavicular and supraclavicular approaches to the brachial plexus using neurostimulation. Korean Journal of Anesthesiology. 2010;58:260.

6. Singh S, Goyal R, Upadhyay K, Sethi N, Sharma R, Sharma A. An evaluation of brachial plexus block using a nerve stimulator versus ultrasound guidance: A randomized controlled trial. J Anaesthesiol Clin Pharmacol.2015;31:370.

7. Winnie AP, Collins VJ. The subclavian perivascular technique of brachial plexus anesthesia. Anesthesiology 1964;25:35-63.

8. Chandrasekaran Ayyavu, Geethanjali Rajamani, Kundhavi Devi, Heber Anandan. A comparative study of brachial plexus block using infraclavicular (coracoid) and axillary approaches in forearm surgery. International Journal of Contemporary Medical Research 
2016;3(9):2507-2510.

9. Susmita Bhattacharyya, KalyanBrata Mandal, Ranabir Chanda, JayantaChakrabarti, Utpal Barman and Debojyoti Das. "A Comparative Study of Interscalene and Supraclavicular Approach of Brachial Plexus Block on Upper Limb Surgeries". EC Anaesthesia 2.5 (2015): 197-204.

10. Whiffler K. Coracoid block - a safe and easy technique. British Journal of Anesthesia. 1981;53:845-8.

11. Desroches J. The infraclavicular brachial plexus block by the coracoid approach is clinically effective: An observational study of 150 patients. Can J Anaesth. 2003;50:253 7.

12. Chin KJ, Alakkad H, Adhikary SD, Singh M Infraclavicular brachial plexus block for regional anesthesia of the lower arm.Cochrane Database Syst Rev. 2013;8:CD005487.

13. Neuburger M, Kaiser H, Rembold-Schuster I, Landes H. Vertical infraclavicular block of the brachial plexus - a clinical study to the reliability of a new method for plexus anesthesia of the upper extremity. Anesthetist. 1998;47:595-9.

14. Kilka HG, Geiger P, Mehrkens HH. Infraclavicular vertical brachial plexus blockade: A new technique of regional anesthesia. Anesthetist. 1995;44:339-44.

15. Ertug Z, Yegin A, Ertem S, Sahin N, Hadimioglu N, Dösemeci L, Erman M. Comparison of two different techniques for brachial plexus block Infraclavicular versus axillary technique. Acta Anaesthesiol Scand. 2005;49:1035-9.

16. Deleuze A, Gentili ME, Marret E, Lamonerie L, Bonnet F. A comparison of a single-stimulation lateral Infraclavicular plexus block with a triple- stimulation axillary block. Reg Anesth Pain Med. 2003;28:89-94.

Copyright: () the author(s), 2020. It is an open-access article distributed under the terms of the Creative Commons Attribution License (CC BY 4.0), which permits authors to retain ownership of the copyright for their content, and allow anyone to download, reuse, reprint, modify, distribute and/or copy the content as long as the original authors and source are cited.

How to cite this article: Mustafa A, Mohammed A, Banu S. A Comparative Study of Different Techniques of Brachial Plexus Block in Upper Limb Surgery. Acad. Anesthesiol. Int. 2020;5(1):05-12.

DOI: dx.doi.org/10.21276/aan.2020.5.1.2

Source of Support: Nil, Conflict of Interest: None declared. 